\title{
Hexagons and spiral defect chaos in non-Boussinesq convection at low Prandtl numbers
}

\author{
Santiago Madruga ${ }^{1}$ and Hermann Riecke ${ }^{2}$ \\ ${ }^{1}$ Max-Planck-Institute for Physics of Complex Systems, D-01187 Dresden, Germany \\ ${ }^{2}$ Department of Engineering Sciences and Applied Mathematics, Northwestern University, Evanston, Illinois 60208, USA
}

(Received 22 December 2005; revised manuscript received 11 October 2006; published 20 February 2007)

\begin{abstract}
We study the stability and dynamics of non-Boussinesq convection in pure gases $\left(\mathrm{CO}_{2}\right.$ and $\left.\mathrm{SF}_{6}\right)$ with Prandtl numbers near $\mathrm{Pr} \simeq 1$ and in a $\mathrm{H}_{2}$-Xe mixture with $\mathrm{Pr}=0.17$. Focusing on the strongly nonlinear regime we employ Galerkin stability analyses and direct numerical simulations of the Navier-Stokes equations. For Pr $\simeq 1$ and intermediate non-Boussinesq effects we find reentrance of stable hexagons as the Rayleigh number is increased. For stronger non-Boussinesq effects the usual, transverse side-band instability is superseded by a longitudinal side-band instability. Moreover, the hexagons do not exhibit any amplitude instability to rolls. Seemingly, this result contradicts the experimentally observed transition from hexagons to rolls. We resolve this discrepancy by including the effect of the lateral walls. Non-Boussinesq effects modify the spiral defect chaos observed for larger Rayleigh numbers. For convection in $\mathrm{SF}_{6}$ we find that non-Boussinesq effects strongly increase the number of small, compact convection cells and with it enhance the cellular character of the patterns. $\mathrm{In}_{2}-\mathrm{Xe}$, closer to threshold, we find instead an enhanced tendency toward roll-like structures. In both cases the number of spirals and of targetlike components is reduced. We quantify these effects using recently developed diagnostics of the geometric properties of the patterns.
\end{abstract}

DOI: 10.1103/PhysRevE.75.026210 PACS number(s): 05.45.Jn, 47.54. $-\mathrm{r}, 47.52 .+\mathrm{j}$, 47.20.Bp

\section{INTRODUCTION}

Rayleigh-Bénard convection of a horizontal fluid layer heated from below is a paradigmatic system for the study of complex spatial and spatio-temporal patterns [1]. Usually, the system parameters are chosen such that variations of the fluid properties across the layer are very small. In the theoretical description this allows the Oberbeck-Boussinesq (OB) approximation, in which the temperature dependence is neglected in all fluid properties except for the density term that is responsible for the buoyancy. For large temperature differences across the layer the variation of the fluid properties with the temperature become significant. These nonOberbeck-Boussinesq (NB) effects break the up-down symmetry that is characteristic of the $\mathrm{OB}$ approximation and, thus, allow a resonant triad interaction among three Fourier modes whose wave vectors form a hexagonal planform. Due to the resonant interaction the primary bifurcation to the hexagons is transcritical, and hexagons are preferred over rolls in the immediate vicinity of onset [2].

Within the framework of the leading-order weakly nonlinear analysis, hexagons typically become unstable to rolls further above threshold, where the amplitudes are larger and the resonant-triad interaction loses significance compared to interactions involving four modes [2-7]. This scenario of a transition from hexagons to rolls has been confirmed in quite a number of experimental investigations [8-13], and quite commonly it has been assumed that hexagon patterns in NB convection are confined to the regime close to onset.

Two convection experiments using $\mathrm{SF}_{6}$ as the working fluid $[14,15]$ have shown, however, that even in the strongly nonlinear regime stable hexagon patterns can be observed. Under OB conditions [14] hexagons were found at relatively high Rayleigh numbers, $\epsilon \equiv\left(R-R_{c}\right) / R_{c} \approx 3.5$ [14]. Due to the mid-plane symmetry of $\mathrm{OB}$ convection hexagons with up-flow in the center coexist stably with down-flow hexa- gons in this regime. In experiments using $\mathrm{SF}_{6}$ under $\mathrm{NB}$ conditions [15] the hexagons that appear at threshold were replaced by rolls for somewhat larger heating and then the hexagons reappeared in the strongly nonlinear regime near $\epsilon=O(1)$. The restabilization was attributed to the large compressibility of $\mathrm{SF}_{6}$ near its critical point [15]. The hexagons that regain stability were termed reentrant hexagons.

Recent numerical computations [16] have demonstrated that hexagons can restabilize in NB convection even if the fluid is incompressible. For instance, in water hexagons that are unstable at $\epsilon=\left(R-R_{c}\right) / R_{c}=0.15$ can already restabilize at $\epsilon=0.2$. The origin of this reentrance was traced back to the existence of stable hexagons in OB convection at large Rayleigh numbers, and the dependence of the NB effects on the Rayleigh number.

At low Prandtl numbers $(\operatorname{Pr} \simeq 1)$ and further above onset OB convection exhibits a new state: spiral defect chaos (SDC). It was first found experimentally [17-19] and then investigated theoretically using simple models $[20,21]$ as well as simulations of the full fluid equations [22,23]. This fascinating state of spatio-temporal chaos is characterized by rotating spirals with varying numbers of arms and of different size, which appear and disappear irregularly and interact with each other and with other defects. SDC arises from the roll state at a threshold that can be as low as $\epsilon=0.1$ in the limit of small Pr. It is driven by large-scale flows that are induced by roll curvature and have a strength that is proportional to $\operatorname{Pr}^{-1}$ [23].

So far, strongly nonlinear NB convection has been studied mostly for large Prandtl numbers [16,24,25], but little is known for small $(\mathrm{Pr} \simeq 1)$ or very small Prandtl numbers $(\operatorname{Pr} \ll 1)$. In particular, whether reentrant hexagons exist at large $\epsilon$ in the presence of the large-scale flows that arise at low Pr, and how NB effects impact spiral defect chaos are interesting questions, which we address in this paper.

Here we study NB convection in gases with small Prandtl numbers. Specifically, we consider parameters corresponding 
to convection in $\mathrm{CO}_{2}$ and $\mathrm{SF}_{6}(\mathrm{Pr} \simeq 0.8)$ and in a $\mathrm{H}_{2}$-Xe mixture $(\mathrm{Pr}=0.17)$. We show that reentrant hexagons are possible in convection in $\mathrm{CO}_{2}$ and confirm the appearance of a longitudinal side-band instability at low wave numbers, which was predicted by weakly nonlinear theory [26]. For spiral defect chaos in $\mathrm{SF}_{6}$ we find that $\mathrm{NB}$ effects promote small convection cells (bubbles). In $\mathrm{H}_{2}-\mathrm{Xe}$, closer to threshold, roll-like structures dominate. In both cases the NB effects reduce the spiral character of the pattern. We quantify the impact of the NB effects on spiral defect chaos using geometric diagnostics that we have proposed recently [27].

The paper is organized as follows. In Sec. II we briefly review the basic equations, emphasizing how our computations focus on weakly non-Boussinesq effects, but strongly nonlinear convection. In Sec. III we present the results for the linear stability of hexagons and rolls in $\mathrm{CO}_{2}$ for a range of parameters accessible experimentally. To compare with experiments, we study the influence of different lateral boundary conditions on the transition from hexagons to rolls in Sec. IV. In Sec. V we discuss spiral defect chaos in $\mathrm{SF}_{6}$ under NB conditions. The stability of hexagons and spiral defect chaos in fluids with very low Prandlt number ( $\mathrm{Pr}$ $=0.17$ ) is studied in a mixture of $\mathrm{H}_{2}$ and Xe in Sec. VI. Finally, conclusions are drawn in Sec. VII.

\section{BASIC EQUATIONS}

The basic equations that we use for the description of NB convection have been discussed in detail previously $[16,24]$. We therefore give here only a brief summary. We consider a horizontal fluid layer of thickness $d$, density $\rho$, kinematic viscosity $\nu$, heat conductivity $\lambda$, thermal diffusivity $\kappa$, and specific heat $c_{p}$. The system is heated from below (at temperature $T_{1}$ ) and cooled from above (at temperature $T_{2}<T_{1}$ ).

To render the governing equations and boundary conditions dimensionless we choose the length $d$, the time $d^{2} / \kappa_{0}$, the velocity $\kappa_{0} / d$, the pressure $\rho_{0} \nu_{0} \kappa_{0} / d^{2}$, and the temperature $T_{s}=\nu_{0} \kappa_{0} / \alpha_{0} g d^{3}$ as the respective scales. The subscripted quantities refer to the respective values at half-depth of the fluid layer in the conductive state. The nondimensionalization gives rise to two dimensionless quantities: the Prandtl number $\operatorname{Pr}=\nu_{0} / \kappa_{0}$, and the Rayleigh number $R$ $=\alpha_{0} \Delta T g d^{3} / \nu_{0} \kappa_{0}$. Furthermore, we write the equations in terms of the dimensionless momentum density $v_{i}$ $=\rho d u_{i} / \rho_{0} \kappa_{0}$ instead of the velocities $u_{i}$. The dimensionless form of the temperature $\hat{T}=T / T_{s}$, heat conductivity $\hat{\lambda}$ $=\lambda / \lambda_{0}$, density $\hat{\rho}=\rho / \rho_{0}$, kinematic viscosity $\hat{\nu}=\nu / \nu_{0}$, and specific heat $\hat{c}_{p}=c_{p} / c_{p 0}$ will be used in the ensuing equations and the hats dropped for clarity. In dimensionless form the equations for the momentum, mass conservation and heat are then given, respectively, by

$$
\begin{aligned}
\frac{1}{\operatorname{Pr}}\left[\partial_{t} v_{i}+v_{j} \partial_{j}\left(\frac{v_{i}}{\rho}\right)\right]= & -\partial_{i} p+\delta_{i 3}\left[1+\gamma_{1}\left(-2 z+\frac{\Theta}{R}\right)\right] \Theta \\
& +\partial_{j}\left\{\nu \rho\left[\partial_{i}\left(\frac{v_{j}}{\rho}\right)+\partial_{j}\left(\frac{v_{i}}{\rho}\right)\right]\right\} \\
& \partial_{j} v_{j}=0
\end{aligned}
$$

$$
\partial_{t} \Theta+\frac{v_{j}}{\rho} \partial_{j} \Theta=\frac{1}{\rho c_{p}} \partial_{j}\left(\lambda \partial_{j} \Theta\right)-\gamma_{3} \partial_{z} \Theta-R \frac{v_{z}}{\rho}\left(1+\gamma_{3} z\right),
$$

with the dimensionless boundary conditions

$$
\vec{v}(x, y, z, t)=\Theta(x, y, z, t)=0 \quad \text { at } z= \pm \frac{1}{2} .
$$

Here $\Theta$ is the deviation of the temperature field from the basic conductive profile. Summation over repeated indices is assumed.

We consider the NB effects to be weak and retain in a Taylor expansion of all material properties only the leadingorder temperature dependence beyond the OB approximation. For the density this implies also a quadratic term with coefficient $\gamma_{1}$. It contributes, however, only to the buoyancy term in (1); in all other expressions it would constitute only a quadratic correction to the leading-order NB effect. Thus, the remaining temperature dependence of the fluid parameters $\rho, \nu, \lambda$, and $c_{p}$ in (1)-(3) is taken to be linear

$$
\begin{aligned}
& \rho(\Theta)=1-\gamma_{0}\left(-z+\frac{\Theta}{R}\right), \\
& \nu(\Theta)=1+\gamma_{2}\left(-z+\frac{\Theta}{R}\right), \\
& \lambda(\Theta)=1+\gamma_{3}\left(-z+\frac{\Theta}{R}\right), \\
& c_{p}(\Theta)=1+\gamma_{4}\left(-z+\frac{\Theta}{R}\right) .
\end{aligned}
$$

The coefficients $\gamma_{i}$ give the difference of the respective fluid properties across the layer. They depend therefore linearly on the Rayleigh number,

$$
\gamma_{i}(\Delta T)=\gamma_{i}^{c}\left(\frac{R}{R_{c}}\right)=\gamma_{i}^{c}(1+\epsilon),
$$

where $\gamma_{i}^{c}$ is the value of $\gamma_{i}$ at the onset of convection and $\epsilon \equiv\left[R-R_{c}\left(\gamma_{i}^{c}\right)\right] / R_{c}\left(\gamma_{i}^{c}\right)$ is the reduced Rayleigh number.

In analogy to Ref. [2], we further omit NB terms that contain cubic nonlinearities in the amplitudes $v_{i}$ or $\Theta$, as they arise from the expansion of the advection terms $v_{j} \partial_{j}\left(v_{i} / \rho\right)$ and $\left(v_{j} / \rho\right) \partial_{j} \Theta$ when the temperature dependence of the density is taken into account. Since we will be considering Rayleigh numbers up to 2 times the critical value, which implies enhanced NB effects, these approximations may lead to quantitative differences compared to the fully NB system, even though the temperature dependence of the material properties themselves is in most situations well described by the linear (or quadratic in the case of the density) approximation.

To quantify the overall strength of the NB effects we use Busse's NB parameter $Q$, which is given by 
TABLE I. Values for the critical temperature (in ${ }^{\circ} \mathrm{C}$ ), the Prandtl number Pr, NB coefficients $\gamma_{i}^{c}$, and Busse's parameter $Q$ for $\mathrm{CO}_{2}$ at the onset of convection for three values of the mean temperature (in ${ }^{\circ} \mathrm{C}$ ). The values correspond to a layer thickness of $d=0.08 \mathrm{~cm}$ and a pressure of $P=300 \mathrm{psi}$.

\begin{tabular}{ccccccccc}
\hline \hline$T_{0}$ & $\Delta T_{c}$ & $\operatorname{Pr}$ & $\gamma_{0}^{c}$ & $\gamma_{1}^{c}$ & $\gamma_{2}^{c}$ & $\gamma_{3}^{c}$ & $\gamma_{4}^{c}$ & $Q$ \\
\hline 20 & 9.43 & 0.87 & 0.0486 & -0.0669 & 0.0779 & 0.0236 & -0.0251 & 1.199 \\
40 & 15.52 & 0.84 & 0.0685 & -0.0883 & 0.1132 & 0.0508 & -0.0184 & 1.724 \\
60 & 23.80 & 0.82 & 0.0931 & -0.1148 & 0.1566 & 0.0919 & -0.0074 & 2.430 \\
\hline
\end{tabular}

$$
Q=\sum_{i=0}^{4} \gamma_{i}^{c} \mathcal{P}_{i},
$$

where the quantities $\mathcal{P}_{i}$ are linear functions of $\operatorname{Pr}^{-1}[1]$. The NB parameter $Q$ quantifies the breaking of the up-down symmetry, which renders at most one of the two types of hexagons stable. Gases have a positive value of $Q$ and exhibit hexagons with down-flow in the center ( $g$-hexagons), whereas liquids have negative $Q$ and show hexagons with up-flow ( $l$-hexagons).

We focus in this paper on the stability properties of patterns in the strongly nonlinear regime. They are determined by a Galerkin method (e.g., Ref. [28]). We use a Fourier expansion on a hexagonal lattice in the lateral directions. The Fourier wave vectors $\mathbf{q}$ are constructed as linear combinations of the hexagon basis vectors $\mathbf{b}_{1}=q(1,0)$ and $\mathbf{b}_{2}$ $=q(1 / 2, \sqrt{3} / 2)$ with $\mathbf{q}=m \mathbf{b}_{1}+n \mathbf{b}_{2}$ where the integers $m$ and $n$ are in the range $\left|m \mathbf{b}_{1}+n \mathbf{b}_{2}\right| \leq n_{q} q$. The largest wave number is then $n_{q} q$ and the number of Fourier modes retained is given by $1+6 \sum_{j=1}^{n_{q} j} j$. Typically we use $n_{q}=3$. The top and bottom boundary conditions are satisfied by using appropriate combinations of trigonometric and Chandrasekhar functions in $z$ [2,29]. In most of the computations we use $n_{z}=6$ modes for each field in Eqs. (1)-(3). The linear analysis yields the critical Rayleigh number $R_{c}$ as well as the critical wave number $q_{c}$. Both depend on the NB coefficients $\gamma_{i}^{c}$ which in turn depend on $R_{c}$. Thus, in principle one obtains an implicit equation for the $\gamma_{i}^{c}$. The shift in the critical Rayleigh number away from the classical value $R_{c}=1708$ due to the NB effects is, however, quite small (less than 1\%) and therefore the resulting change in the $\gamma_{i}^{c}$ is negligible. In this paper we therefore choose the $\gamma_{i}^{c}$ corresponding to $R_{c}=1708$.

To investigate the nonlinear hexagon solutions, we start with the standard weakly nonlinear analysis to determine the coefficients of the three coupled amplitude equations for the modes making up the hexagon pattern. To obtain the fully nonlinear solutions requires the solution of a set of nonlinear algebraic equations for the expansion coefficients with respect to the Galerkin modes. This is achieved with a Newton solver for which the weakly nonlinear solutions serve as convenient starting solutions. In the Galerkin code amplitude instabilities are tested by linear perturbations of the expansion coefficients. In addition, modulational instabilities are considered, which involve the introduction of Floquet multipliers $\exp (i \mathbf{s} \cdot(x, y))$ in the Fourier ansatz for the linear perturbations of the Galerkin solutions.

We also study the temporal evolution of the system. For that we employ a Fourier spectral code on a rectangular grid $\left(i d q_{x}, j d q_{y}\right), i, j=1, \ldots, N$, with $d q_{y} / d q_{x}=\sqrt{3} / 2$ to accommodate perfect hexagonal patterns. The same vertical modes are used as in the Galerkin stability code [22]. To solve for the time dependence a fully implicit scheme is used for the linear terms, whereas the nonlinear parts are treated explicitly (second order Adams-Bashforth method). The time step is typically taken to be $t_{v} / 500$, where $t_{v}$ is the vertical diffusion time. We have tested that the stability regimes obtained from the Galerkin analysis are consistent with the direct numerical simulations. Both codes employed in this paper have been kindly provided by Pesch [22,30].

\section{REENTRANT HEXAGONS IN $\mathrm{CO}_{2}$}

In this paper we investigate specific scenarios for convection in gases that should be experimentally realizable. We focus in this section on $\mathrm{CO}_{2}$ in a conventional range for the layer thickness, pressure, and temperature. Table I provides the NB coefficients and the $Q$ value at the onset of convection for a representative range of the mean temperature $T_{0}$ in a layer of thickness $d=0.08 \mathrm{~cm}$ [57].

\section{A. Amplitude instabilities}

In our analysis we first concentrate on spatially periodic solutions with their wave number being fixed at the critical wave number and discuss their domains of existence and their stability with respect to amplitude instabilities, which do not change the wave number. For three different cells with thicknesses $d=0.07,0.08$, and $0.09 \mathrm{~cm}$, respectively, we consider the range $0<\epsilon<1$ at a pressure of $P=300$ psi.

The results of the stability analysis for hexagons and rolls are shown in Fig. 1. The hexagons are linearly stable for very small $\epsilon$. For a given layer thickness and not too high mean temperature $T_{0}$ the hexagons become unstable as the control parameter is increased. The hexagon patterns then undergo a second steady bifurcation as the control parameter is increased further and become stable again. Such restabilized hexagons have been termed reentrant hexagons [14-16]. As the mean temperature is increased or the layer thickness is decreased the critical heating and with it the NB effects increase. This shifts the point of reentrance to lower $\epsilon$ and the lower stability limit to higher $\epsilon$, decreasing the $\epsilon$-range over which the hexagons are unstable, until the two limits merge at a temperature $T_{m}$. For $T_{0}>T_{m}$ the hexagons are amplitudestable over the whole range of $\epsilon$ considered $(0 \leq \epsilon \leq 1)$.

We have also computed the stability of rolls with respect to amplitude perturbations. The corresponding stability limits are indicated in Fig. 1 by thin lines. Rolls are stable above 


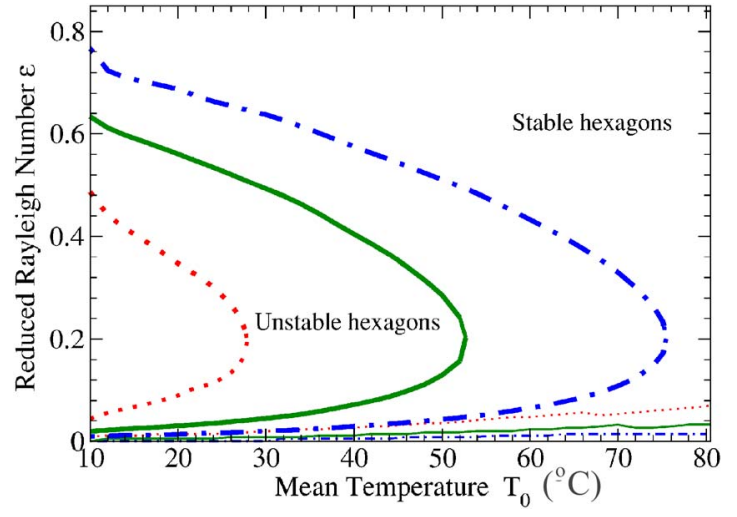

FIG. 1. (Color online) Stability regions for hexagons and rolls in $\mathrm{CO}_{2}$ with respect to amplitude perturbations for three fluid depths: $d=0.07 \mathrm{~cm}$ (dotted lines), $d=0.08 \mathrm{~cm}$ (full lines), $d=0.09 \mathrm{~cm}$ (dotted-dashed line). Pressure is kept at $P=300$ psi. Thick lines, stability boundaries for hexagons. Thin lines, stability boundaries for rolls. For a given depth, rolls are stable above the thin line, and hexagons are unstable in the inner region of the thick line.

these lines. As the NB effects become stronger the stabilization of rolls is shifted to larger $\epsilon$. In contrast to the hexagons, the rolls do not undergo a second bifurcation within the parameter regime investigated and remain amplitude-stable beyond $\epsilon=1.0$. For strong NB effects one has therefore a large range of parameters over which the competing rolls and hexagons are both linearly amplitude stable.

The amplitude-stability limits of the hexagons and rolls depend on their wave number. This is illustrated for the hexagons in Fig. 2 for a mean temperature of $T_{0}=40^{\circ} \mathrm{C}$. The instability region with respect to amplitude perturbations forms a bubblelike closed curve, inside of which the hexagons are unstable with respect to amplitude perturbations.

It is worth mentioning that the stability limits for hexagons in $\mathrm{CO}_{2}$ are quite similar to those of NB convection in

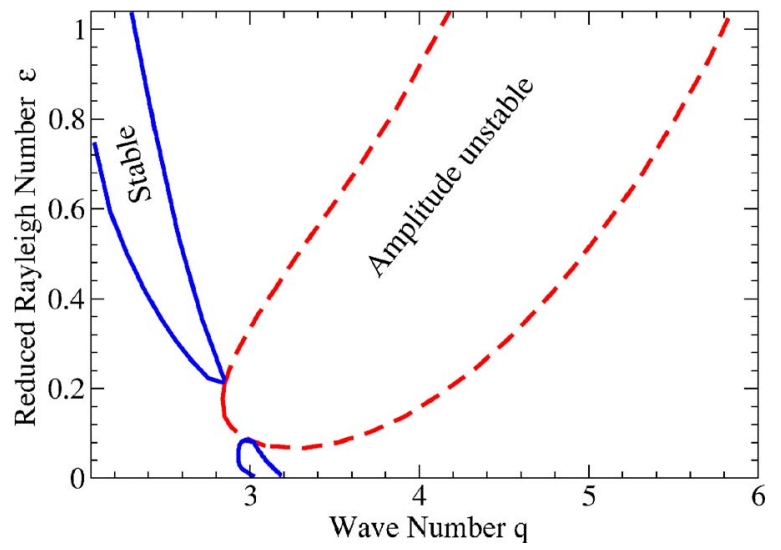

FIG. 2. (Color online) Stability regions for hexagons in $\mathrm{CO}_{2}$ with respect to amplitude (dashed line) and side-band perturbations (solid line) for layer depth $d=0.08 \mathrm{~cm}$, pressure $P=300 \mathrm{psi}$, mean temperature $T_{0}=40{ }^{\circ} \mathrm{C}$, and Prandtl number $\operatorname{Pr}=0.84$. The NB coefficients $\gamma_{i}^{c}$ are reported in Table I. Hexagons are stable with respect to amplitude perturbations outside the dashed-line region, and stable with respect to side-band perturbation inside the solid-line region.

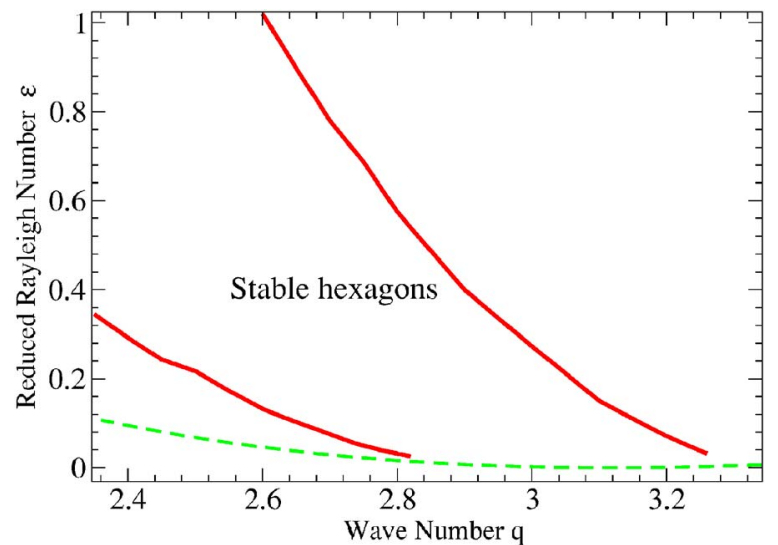

FIG. 3. (Color online) Stability regions for hexagons in $\mathrm{CO}_{2}$ with respect to side-band perturbations for layer thickness $d$ $=0.052 \mathrm{~cm}$, pressure $P=300 \mathrm{psi}$, mean temperature $T_{0}=27^{\circ} \mathrm{C}$, and Prandtl $\operatorname{Pr}=0.86$. The NB coefficients are $\gamma_{0}^{c}=0.2053, \quad \gamma_{1}^{c}$ $=-0.2877, \gamma_{2}^{c}=0.3267, \gamma_{3}^{c}=0.1152$, and $\gamma_{4}^{c}=-0.086$. The hexagons are stable with respect to side-band perturbations in the region inside the solid lines and unstable outside. The dashed line corresponds to the neutral curve.

water, except that in $\mathrm{CO}_{2}$ the $\mathrm{NB}$ effects increase rather than decrease with increasing mean temperature [16].

\section{B. Side-band instabilities}

In systems with sufficiently large aspect ratio side-band instabilities can be the most relevant instabilities. Using the Galerkin method, we have studied the stability of the hexagons with respect to long- and short-wave side-band perturbations for $T_{0}=40^{\circ} \mathrm{C}$. The results are shown in Fig. 2. We find that over the whole range $0 \leq \epsilon \leq 1$ the only relevant side-band perturbations are long-wave and steady, as is the case in the weakly nonlinear regime. The same is true also in water with higher Prandtl number [16]. In this parameter regime the stability region consists of two disconnected domains, reflecting the reentrant nature of the hexagons. The stability domain near onset is very small and closes up as the amplitude-stability limit is reached. In the reentrant regime the stable domain opens up again in an analogous fashion when the amplitude-stability limit is crossed. Note that the stability boundaries are leaning toward lower wave numbers. Thus, stable reentrant hexagon patterns are expected to have wave numbers below $q_{c}$. This is related to the fact that in the OB case hexagons can be stable for large $\epsilon$, but they are side-band stable only for small wave numbers [31].

As the mean temperature is increased the bubble of the amplitude instability shrinks and eventually disappears, as shown in Fig. 3 for a cell with thickness $d=0.052 \mathrm{~cm}$ and mean temperature $T_{0}=27^{\circ} \mathrm{C}$. As before, the relevant sideband instabilities are long wave all along the stability limits and with increasing $\epsilon$ the wave number range over which the hexagons are stable shifts towards smaller wave numbers. For these parameters the region of side-band-stable hexagons reaches without interruption from the strongly nonlinear regime all the way down to threshold. For yet stronger NB effects the range of stable wave numbers widens. 

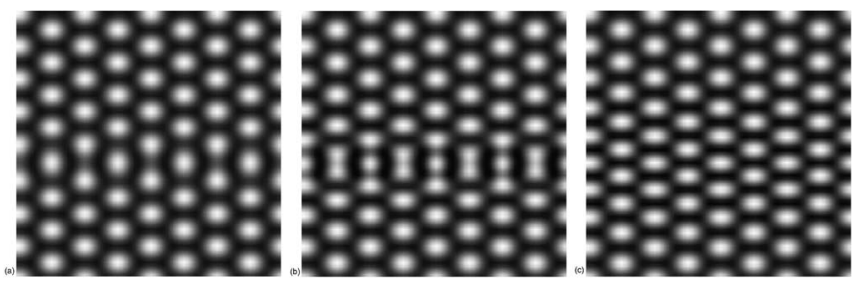

FIG. 4. Longitudinal long-wave instability mode for $q=2.5$ and $\epsilon=0.195$ at times $t=508.2 t_{v}, 514.8 t_{v}$, and $520 t_{v}$ for parameters corresponding to Fig. 3. New cells are created in the region of expansion along a line in the center of the cell. The final state, reached after further relaxation, consists of a regular pattern with spatially homogeneous wave number.

In the weakly nonlinear regime the effect of mean flows on the side-band instabilities of hexagonal convection at low Prandtl numbers has been studied using suitably extended coupled Ginzburg-Landau equations [26] to capture the relevant nonlocal mean-flow effects [32,33]. It was found that in the presence of the mean flow the side-band instabilities remain long wave and that the mean flow affects only the transverse long-wave mode, which leads to a shearing of the pattern, whereas the longitudinal long-wave mode, which induces a compression or expansion of the pattern, is unaffected. In the absence of the mean flow the longitudinal instability is usually relevant only in a very small parameter regime that is even difficult to probe in numerical simulations [34]. For sufficiently low Prandtl numbers the weakly nonlinear theory predicts, however, that on the low- $q$ side the longitudinal instability should replace the usual transverse instability [26]. In numerical simulations of the extended Ginzburg-Landau equations the transient patterns arising from the longitudinal instability were noticeably more ordered than those arising from the transverse one [26].

Inspecting the eigenvectors of the destabilizing modes obtained in the full Galerkin stability analysis we find that for the parameters corresponding to a layer thickness of $d$ $=0.052 \mathrm{~cm}$ (cf. Fig. 3) the destabilizing mode at the high- $q$ side is the usual transverse mode, i.e., the spatial shift induced by the translation mode is perpendicular to its modulation wave vector. At the low- $q$ side, however, the longitudinal mode takes over. Thus, while for $q=3.1, \epsilon=0.160$ in a system of size $8 \times 2 \pi / q$ the maximal growth rate for the transverse mode is +0.005 , it is -0.19 for the longitudinal mode. Conversely, for $q=2.5, \epsilon=0.196$ in the same system size the maximal growth rate of the transverse mode is -0.065 while that for the longitudinal mode is +0.0004 .

To study the evolution ensuing from the two long-wave instabilities we perform direct simulations of the NavierStokes equations (1)-(4) in a cell of size $L=8 \times 2 \pi / q_{c}$ $=16.11$ using $128 \times 128$ Fourier modes. More precisely, to allow perfect hexagons we use a rectangular domain with $L_{x}=L$ and $L_{y}=\sqrt{3} L / 2$. Snapshots of the evolution are shown in Figs. 4 and 5. The longitudinal instability initially leads to a localized expansion of the pattern along a line near the center of the system [Fig. 4(a)]. New cells arise then in this region [Figs. 4(b) and 4(c)], which subsequently diffusively expand. Eventually the system relaxes to an ordered pattern with spatially homogeneous wave number. By contrast, the

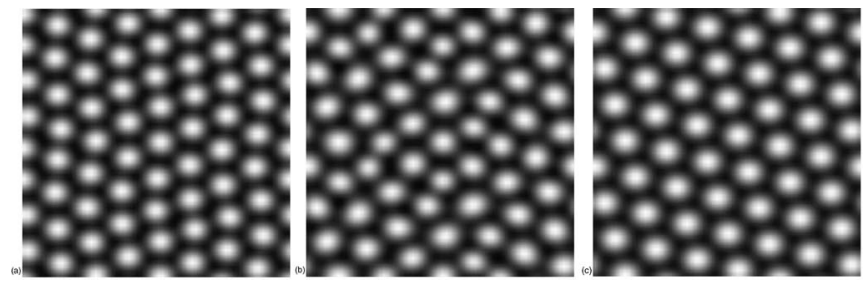

FIG. 5. Transverse long-wave instability mode for $q=3.1$ and $\epsilon=0.16$ at times $t=977.4 t_{v}, 1094.2 t_{v}$, and $1308 t_{v}$ for parameters corresponding to Fig. 3. The transient pattern (b) is substantially more disordered than in the case of the longitudinal mode shown in Fig. 4. The final state is rotated relative to the initial pattern.

modulated shearing arising from the transverse instability for $q=3.1$ [Fig. 5(a)] leads transiently to a quite disordered pattern [Fig. 5(b)] as was suggested by the simulations of the corresponding Ginzburg-Landau equations [26]. Eventually the pattern also relaxes to a perfectly ordered pattern, which is, however, rotated with respect to the initial pattern [Fig. $5(\mathrm{c})]$.

\section{COMPARISON WITH EXPERIMENTS IN $\mathrm{CO}_{2}$}

Bodenschatz et al. [12] carried out a set of experiments on convection in $\mathrm{CO}_{2}$ in a cylindrical cell with aspect ratio $\Gamma$ $\sim 172$, thickness $d=0.052 \mathrm{~cm}$, and pressure $P=300$ psi. Under these conditions NB effects are relevant. In the experiments a weakly hysteretic transition from hexagons to rolls was found near $\epsilon=0.1$. Noting that this transition point was-according to weakly nonlinear theory-below the amplitude instability of hexagons to rolls, the authors interpreted their results in terms of the heterogeneous nucleation of rolls by the sidewalls. They found that for small $\epsilon$ the concentric rolls induced by the sidewall heating remained confined to the immediate vicinity of the sidewalls; however, as $\epsilon$ was increased the rolls invaded the hexagons and filled the whole cell, inducing a transition from hexagons to rolls.

A comparison of the experimental findings with the stability results shown in Fig. 3 shows that indeed the transition cannot be due to an amplitude instability of the hexagons. In fact, in this regime the NB effects are so strong that, in contrast to the predictions of the weakly nonlinear theory, the hexagons do not undergo an amplitude instability at all. To clarify the influence of the sidewalls and to assess the significance of the side-band instabilities for the transition from hexagons to rolls, we perform direct simulations of the Navier-Stokes equations (1)-(4) for two different sets of boundary conditions [58].

(i) Periodic boundary conditions. According to Fig. 3, for $\epsilon=0.3$ hexagons with wave numbers larger than $q>2.98$, which includes the critical wave number, are unstable with respect to side-band instabilities. To test whether these sideband instabilities trigger a transition to rolls we perform numerical simulations with periodic boundary conditions and hexagons as initial conditions. Figure 6 presents some snapshots of the ensuing temporal evolution in a cell of size $L$ $=8 \times 2 \pi / q_{c}=16.11$ using again $128 \times 128$ Fourier modes. The sideband instability of the initially almost perfect hexa- 

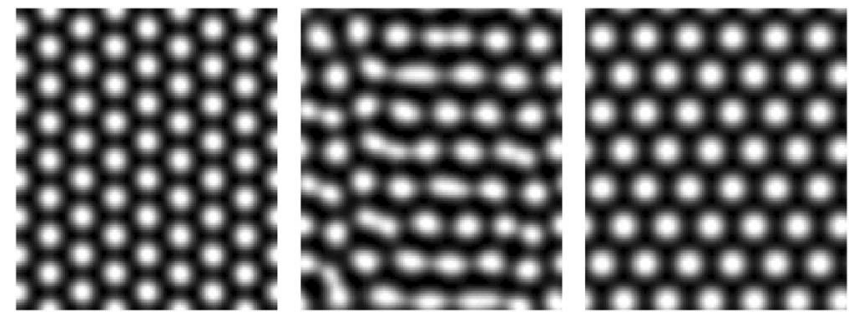

FIG. 6. Numerical simulation corresponding to a layer of $\mathrm{CO}_{2}$ with thickness $d=0.052 \mathrm{~cm}$, pressure $P=316 \mathrm{psi}$, mean temperature $T_{0}=27.3^{\circ} \mathrm{C}$, Prandtl number $\operatorname{Pr}=0.87$, and control parameter $\epsilon$ $=0.3$ (cf. Fig. 3). The size of the integration domain is $L=8$ $\times 2 \pi / q_{c}=16.11$ and the boundary conditions are periodic. As initial condition a slightly perturbed hexagon pattern with wave number $q_{c}=3.12$ has been used. (a) corresponds to $t=0$, (b) to $t=18 t_{v}$, and (c) to $t=60 t_{v}$.

gon pattern [cf. 6(a)] induces a shearing of the pattern [cf. 6(b)]. At the same time a few penta-hepta defects arise and some hexagonal convection cells tend to connect with each other forming short roll-like structures. However, as time evolves the system becomes progressively more ordered again and eventually, after losing a number of convection cells, a defect-free hexagon pattern with a smaller wave number is established [cf. 6(c) at $t \simeq 60 t_{v}$ ].

Thus, while roll-like features appear transiently for this value of $\epsilon$, with periodic boundary conditions no transition to rolls occurs and the system relaxes to a new ordered hexagon pattern. Only for yet larger values of $\epsilon$ the roll-like structures that arise in the intermediate, disordered state take over and lead to a transition to rolls induced by the side-band instabilities.

(ii) Concentric rolls as boundary conditions. Clearly, the simulations for periodic boundary conditions do not match the experimental results described above, where a transition from hexagons to rolls occurs already for $\epsilon \gtrsim 0.11$. To address this disagreement we take into account the fact that in the experiments side-wall heating promoted the nucleation of rolls [12]. Strictly speaking, the code we are using does not allow nonperiodic boundary conditions. To mimic the experimentally used cylindrical cell we employ a step ramp in $\epsilon$ that reduces $\epsilon$ to values well below $\epsilon=0$ outside a circle of radius $r=0.45 L$ with $L=16 \times 2 \pi / q_{c}=32.22$ [22]. To induce concentric rolls near the sidewalls we introduce for $r$ $>0.45 \mathrm{~L}$ an additional heating in the interior of the fluid in the form of concentric rings. Using hexagonal initial conditions in the bulk, this leads to an initial state as shown in Fig. 7(a).

Figures 7(a) and 7(b) shows two snapshots at $t=t_{v}$ and $t$ $=158 t_{v}$ demonstrating how the rolls induced by the side walls invade the carefully prepared hexagonal state in the bulk already for $\epsilon=0.2$. This is well below the $\epsilon$ value used in Fig. 6, where with periodic boundary conditions the hexagons persisted even through the side-band instability. For nonperiodic boundary conditions the final, steady state consists of concentric rolls as observed in the experiments (cf. Fig. 5 in Ref. [12]). For lower $\epsilon$, however, the experimentally observed final state consists of hexagons in the bulk of the system surrounded by wall-induced concentric rolls (cf. Fig.

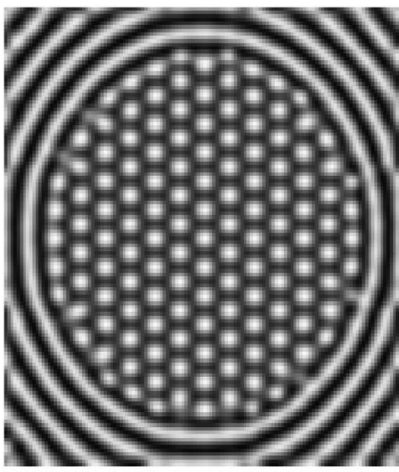

(a)

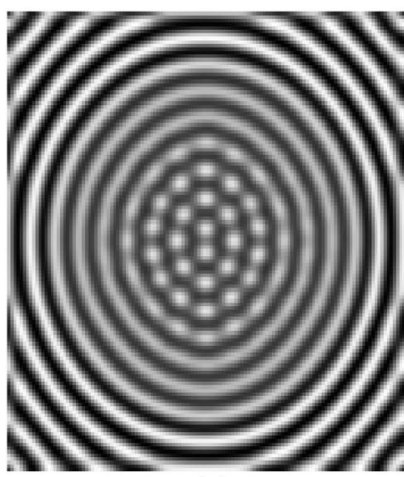

(c)

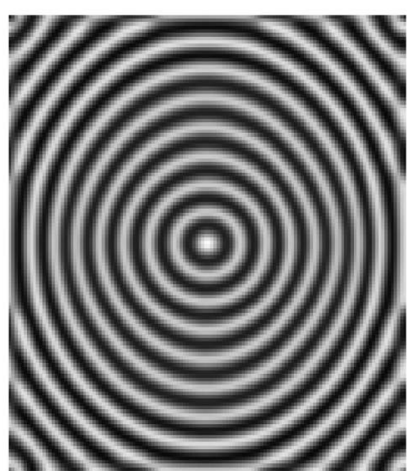

(b)

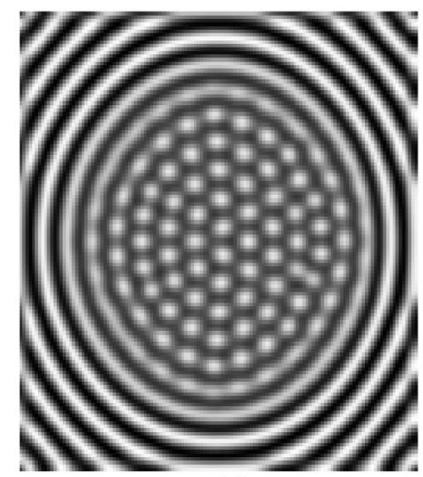

(d)
FIG. 7. Numerical simulation corresponding to a cell of $\mathrm{CO}_{2}$ with thickness $d=0.052 \mathrm{~cm}$, pressure $P=300 \mathrm{psi}$, mean temperature $T_{0}=27{ }^{\circ} \mathrm{C}$, and Prandtl number $\operatorname{Pr}=0.86$. The size of the integration domain is $L=16 \times 2 \pi / q_{c}=32.22$, and the boundary conditions are rings of concentric rolls generated by external forcing. For $\epsilon=0.2$ (upper snapshots) hexagon initial conditions have been used, with $t=t_{v}$ (left snapshot) and $t=158 t_{v}$ (right snapshot). The lower snapshots show a simulation for $\epsilon=0.05$ and random initial conditions, at $t=40 t_{v}$ (left snapshot) and $t=158 t_{v}$ (right snapshot).

4 in Ref. [12]). We find this also in our numerical simulations, as shown in Figs. 7(c) and 7(d). There the forcing of rolls is identical to that in Figs. 7(a) and 7(b) but $\epsilon=0.05$. Starting with random initial conditions, the forcing gives rise to a ring contained in the square integration domain. At the beginning of the simulation [Fig. 7(c)] the rolls created by the forcing invade the interior of this small system. However, as time progresses the rolls pull out of the central region of the cell, and the final steady state (for $t \gtrsim 165 t_{v}$ ) consists of stable hexagons surrounded by a couple of concentric rolls in addition to those induced by the forcing.

Thus, our simulations suggest that the experimentally observed transition from hexagons to rolls is neither due to amplitude instabilities nor to side-band instabilities. Rather, there is a large range of parameters in which hexagons and rolls are both linearly stable and the final state is selected by one type of pattern invading the other. The transition to rolls at these low values of $\epsilon$ is made possible by the boundaries, which provide a seed for the nucleation of rolls. We expect that by applying a forcing that is confined to the region near the boundaries and that replaces the rolls by hexagons the transition to rolls could be shifted to substantially larger values of $\epsilon$. Such a forcing could be achieved by a patterned 
heating of the interior of the fluid [35] or possibly by a suitable geometric patterning of the bottom plate [36].

\section{NON-OBERBECK-BOUSSINESQ SPIRAL DEFECT CHAOS IN SF 6}

A fascinating state observed in convection at low Prandtl numbers is spiral defect chaos. It is characterized by selfsustained chaotic dynamics of rotating spirals, as well as dislocations and disclinations, and arises in fluids with $\mathrm{Pr}$ $\$ 1[17,19-22]$ in a parameter range where straight rolls are linearly stable. Spiral defect chaos has so far predominantly been investigated under $\mathrm{OB}$ conditions, in which up-flows and down-flows are equivalent.

As mentioned before, NB effects break the up-down symmetry and different flow structures may be predominantly associated with up-flow and down-flow, respectively. Moreover, in the absence of the OB symmetry a resonant triad interaction is allowed. If it is strong enough it leads to the formation of hexagons. For weaker interaction one may still expect an enhancement of cellular rather than roll-like structures.

To investigate the impact of NB effects on spiral defect chaos we consider convection in a layer of $\mathrm{SF}_{6}$. This gas has been used previously in experimental convection studies under usual laboratory conditions [37], and near the thermodynamical critical point $[14,15,38]$. In Fig. 8(a) we present the stability diagram for hexagons and rolls with respect to amplitude perturbations in a layer of $\mathrm{SF}_{6}$ of thickness $d$ $=0.0542 \mathrm{~cm}$, pressure $P=140 \mathrm{psi}$, and a range of temperatures that is experimentally accessible. Hexagons are amplitude stable to the right of the solid line and rolls above the dashed line. As in the case of $\mathrm{CO}_{2}$ the $\mathrm{NB}$ effects increase with increasing mean temperature $T_{0}$ and above a certain value of $T_{0}$ hexagons are linearly amplitude stable over the whole range of $\epsilon$ investigated. Here we focus on relatively strong NB effects. We therefore show in Fig. 8(b) the stability limits with respect to side-band perturbations for a relatively large mean temperature, $T_{0}=80^{\circ} \mathrm{C}$. As in the case of $\mathrm{CO}_{2}$, the wave number range over which the hexagons are stable is leaning towards smaller wave numbers. Overall, amplitude and side-band stability limits are qualitatively similar to those of convection in $\mathrm{CO}_{2}$ (cf. Fig. 1).

Figure 9 shows two snapshots obtained by direct numerical simulations of the Navier-Stokes equations corresponding to convection in $\mathrm{SF}_{6}$ for $T_{0}=80{ }^{\circ} \mathrm{C}$ and $\epsilon=1.4$ in a convective cell of thickness $d=0.0542 \mathrm{~cm}$ and horizontal size $L$ $=16 \times 2 \pi / q_{c}=32.22$. Periodic boundary conditions are used with $128 \times 128$ Fourier modes and six vertical modes. Both states are obtained after an integration time of $160 t_{v}$, starting from random initial conditions. While in Fig. 9(a) all NB effects are retained, in Fig. 9(a) the same values are used for Pr and $\epsilon$, but all NB parameters $\gamma_{i}^{c}$ are set to 0, i.e., the system is treated as if it was Boussinesq.

The snapshots depicted in Fig. 9 show, as expected, that due to the NB effects down-flow convection cells, which are white in Fig. 9, outnumber cells with up-flow (black). Moreover, in this regime the NB effects enhance the overall cellular rather than roll-like character of SDC. This manifests
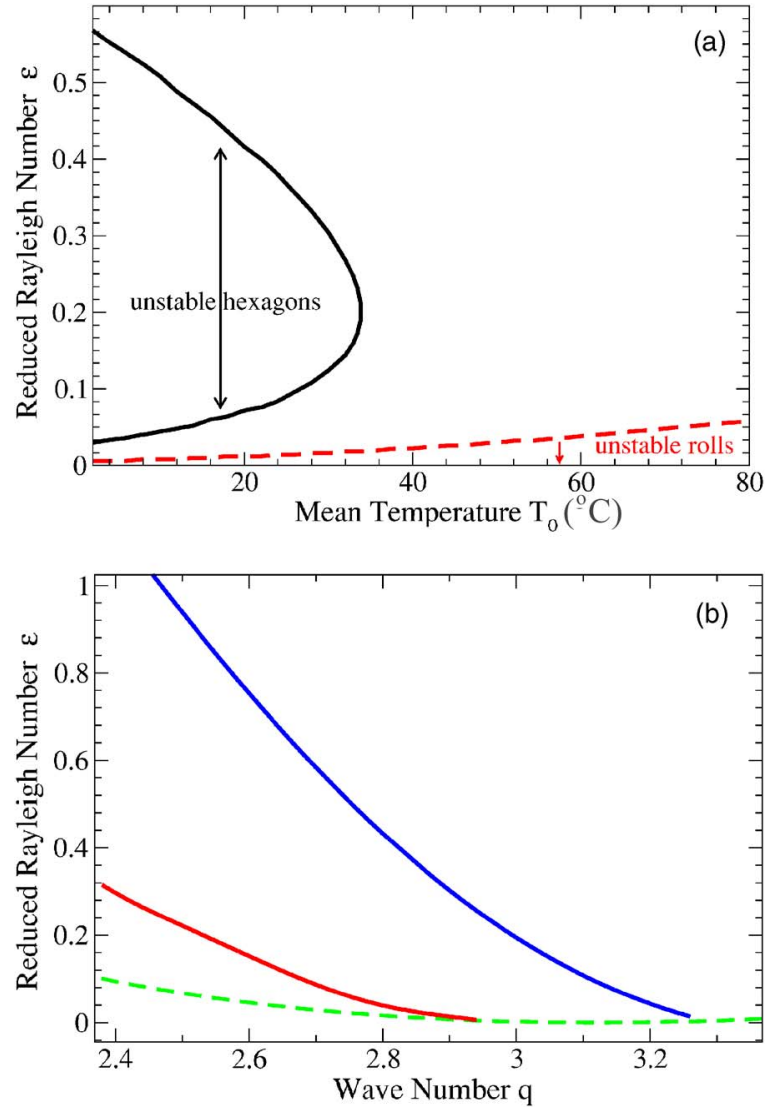

FIG. 8. (Color online) Stability regions of hexagons and rolls in a layer of $\mathrm{SF}_{6}$ of thickness $d=0.0542 \mathrm{~cm}$, pressure $P=140 \mathrm{psi}$, and Prandtl number $\operatorname{Pr}=0.8$. (a) Stability regions with respect to amplitude perturbations. Continues line, stability boundary for hexagons. Dashed line, stability boundary for rolls. Stability limits obtained for the critical wave number $q_{c}$. (b) Stability regions with respect to side-band perturbations for the above layer with a mean temperature $T_{0}=80^{\circ} \mathrm{C}$. The corresponding NB coefficients are $\gamma_{0}^{c}=0.1714$, $\gamma_{1}^{c}=-0.2118, \gamma_{2}^{c}=0.2836, \gamma_{3}^{c}=0.1905$, and $\gamma_{4}^{c}=0.0624$ corresponding to $Q=4.2$. The dashed line corresponds to the neutral curve.

itself in the appearance of numerous small down-flow convection cells (white "bubbles") and in the appearance of quite noticeable bulges on the NB convection rolls. To quantify these and other differences we analyze a long sequence of snapshots with a recently introduced geometric approach [27]. It is based on the contour lines corresponding to the intensity half-way between the minimal and maximal intensity of all snapshots in a given run. The contour lines corresponding to the temperature field of snapshots Figs. 9(a) and 9(b) are shown in Figs. 9(c) and 9(d). In the following we present various statistics of these contour lines.

The most striking difference between the $\mathrm{OB}$ and the NB case is the asymmetry that is induced by the NB effects between "black" and "white" components, i.e., between closed contours that enclose up- and down-flow regions, respectively. To quantify this asymmetry we measure the number of white and black components. These two topological measures correspond, respectively, to the Betti numbers of order 1 and 2 of the pattern defined by the white components [39]. Figure 10 shows these two quantities as a function of 


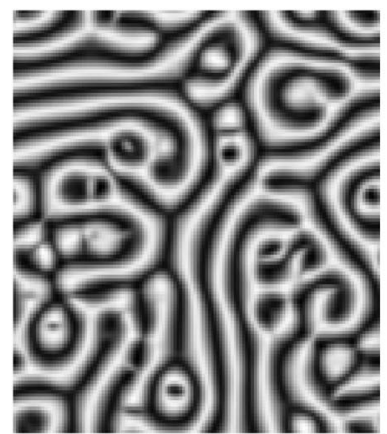

(a)

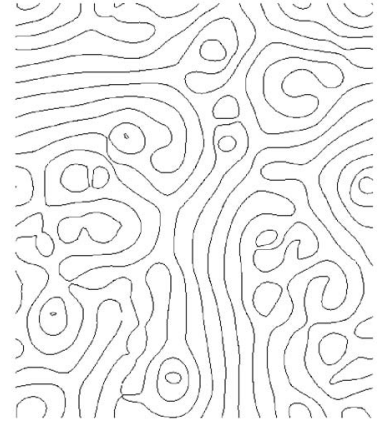

(b)

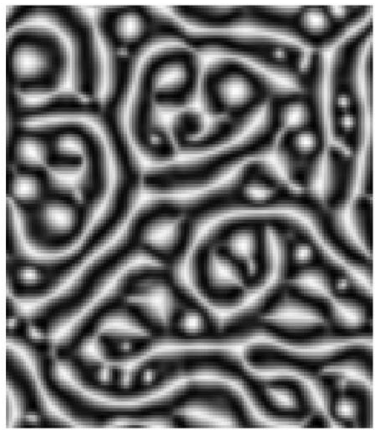

(c)

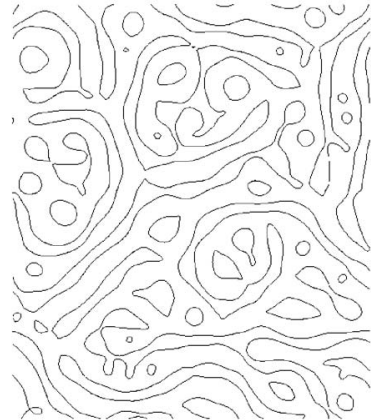

(d)
FIG. 9. Direct numerical simulation of equations (1)-(4) for $\mathrm{SF}_{6}$ in a cell of thickness $d=0.0542 \mathrm{~cm}$, pressure $P=140 \mathrm{psi}$, mean temperature $T_{0}=80{ }^{\circ} \mathrm{C}$, Prandtl number $\operatorname{Pr}=0.8$, and control parameter $\epsilon=1.4$. The cell has size $L=16 \times 2 \pi / q_{c}=32.22$ with periodic boundary conditions. Starting from random initial conditions both snapshots are taken at $t=160 t_{v}$. Left-hand panels for OB conditions $\left(\gamma_{i}=0, i=0 . .4\right.$, left), right-hand panels for NB conditions appropriate for $T_{0}=80^{\circ} \mathrm{C}$ [cf. Fig. 8(b)]. Bottom panels give the corresponding contour lines used for the pattern diagnostics.

time in the $\mathrm{OB}$ and the NB case. As expected, in the OB case the number of black and white components is essentially the same at all times, whereas in the NB case the white components significantly outnumber the black ones. The ratio of white to black components is therefore a sensitive indicator for the significance of NB effects. Figure 10 also illustrates how much the number of components fluctuates during these runs. Recently, the two Betti numbers have also been mea-

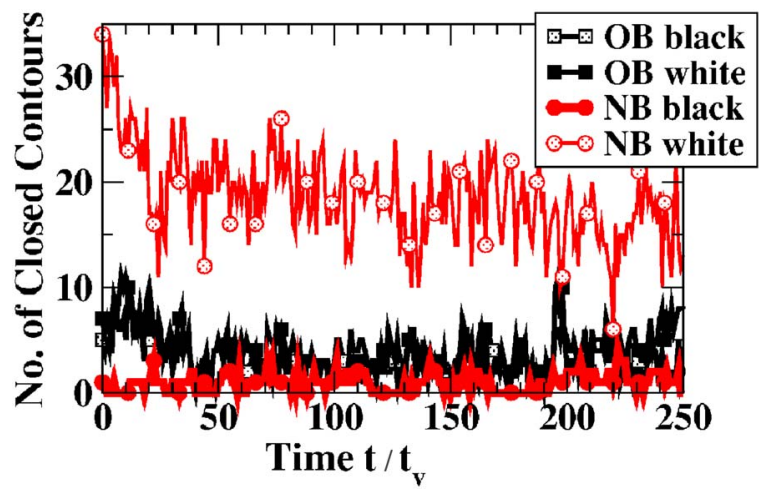

FIG. 10. (Color online) Number of black and white closed contours as a function of time for $\mathrm{NB}$ and $\mathrm{OB}$ conditions for the simulations corresponding to the snapshots shown in Fig. 9.

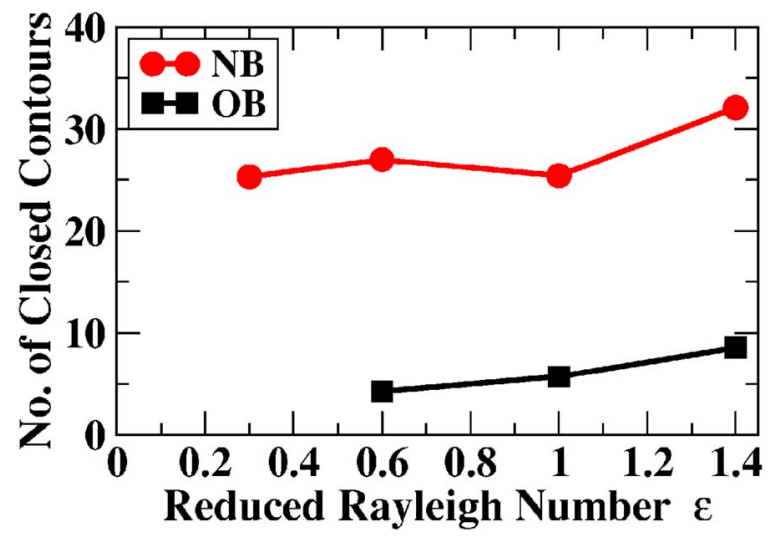

FIG. 11. (Color online) Total number of closed contours as a function of the control parameter $\epsilon$ for NB (circles) and $\mathrm{OB}$ (squares) conditions (cf. Fig. 9).

sured based on patterns obtained in experiments on SDC in convection in $\mathrm{CO}_{2}$. Scanning $\epsilon$ in very small steps the authors report steps in the Betti numbers indicative of transitions between different chaotic, disordered states [40].

Figure 10 shows that the total number of components (closed contours) is considerably larger in the NB case than in the OB case. This is presented in more quantitative detail in Fig. 11, which gives the mean value of the total number of components, i.e., the sum of black and white components, as a function of $\epsilon$ for $\mathrm{OB}$ as well as NB conditions. In the NB case the total number of components is up to 4 times larger than in the OB case. We attribute this difference to the resonant triad interaction that is made possible by the breaking of the OB symmetry and which tends to enhance cellular rather than filamentary roll-like structures.

To characterize the components better and to distinguish cellular and roll-like structures we introduce the compactness $\mathcal{C}$ of components [27],

$$
\mathcal{C}=4 \pi \frac{\mathcal{A}}{\mathcal{P}^{2}} .
$$

Here $\mathcal{A}$ is the area inside a closed contour and $\mathcal{P}$ its perimeter. With the normalization used in (11) compact, cellular structures are characterized by $\mathcal{C} \leqq 1$, whereas filamentary, roll-like structures have $\mathcal{C} \ll 1$.

Figure 12 shows the distribution function for the mean number of closed contours per snapshot as a function of compactness $\mathcal{C}$ for the $\mathrm{NB}$ and the OB simulation at $\epsilon=1.4$ over the duration $t=360 t_{v}$. As expected, in the NB case the number of white components is much larger than that of black components, whereas in the OB case both are about the same. The total number of components is noticeably larger in the NB case, which also shows an increase in the number of white, filamentary contours with small compactness.

The relative distribution among components with different compactness is more clearly visible in the relative frequency of contours as a function of the compactness, which is shown in Fig. 13. More precisely, its data result from running averages over 10 adjacent points of the corresponding data shown in Fig. 12, which are then normalized to give 


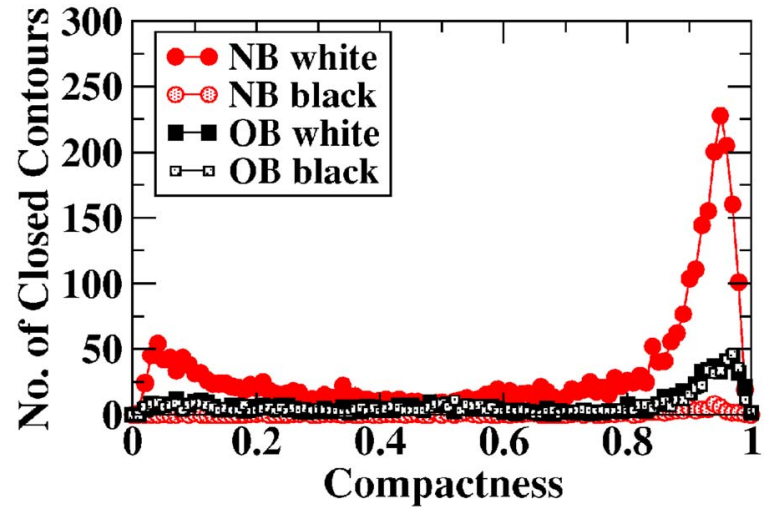

FIG. 12. (Color online) Distribution function for the mean number of closed contours per snapshot as a function of compactness $\mathcal{C}$ for convection in $\mathrm{SF}_{6}$ at $\epsilon=1.4$ under $\mathrm{NB}$ (circles) and under $\mathrm{OB}$ (squares) conditions (cf. Fig. 9).

a unit integral. The normalized data show that the increase in the relative frequency of white filamentary components $(\mathcal{C} \ll 1)$ is essentially the same in the NB case and in the OB case. However, only very few black filamentary components arise in the NB case.

A feature of Fig. 13 that is surprising at first sight is the essentially equal height of the NB peak and the OB peak for components with $\mathcal{C} \sim 1$. Visually, the NB snapshot exhibits many more small compact "bubbles" than the OB run. An explanation for this observation can be obtained by correlating the compactness of the closed contours with their length $\mathcal{P}$. The joint distribution function for these two quantities is shown in Fig. 14 using logarithmic scales for $\mathcal{P}$ and $\mathcal{C}$. Focussing on the compact objects with $\mathcal{C} \lesssim 1$ one recognizes in the $\mathrm{OB}$ case a second peak at somewhat larger contour length. We associate this peak with the appearance of targetlike structures, i.e., with a second contour line encircling a smaller compact, almost circular contour line. In the NB case this second peak is barely visible. Instead, the shoulder of the main peak is extended significantly towards smaller contour length $\mathcal{P}$. It signifies the appearance of compact objects that are smaller than a typical wavelength, which we associate with the small "bubbles" that are easily recognized in the

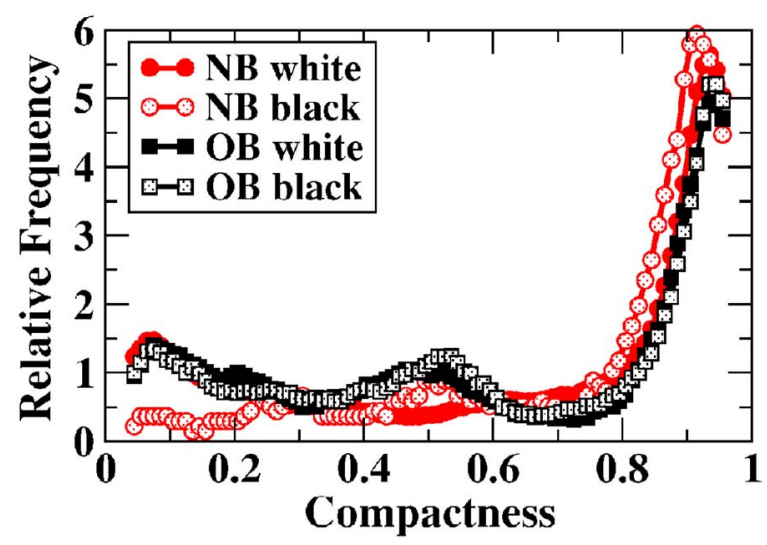

FIG. 13. (Color online) Normalized distribution function for the compactness $\mathcal{C}$ for NB (circles) and OB (squares) conditions (cf. Fig. 9).
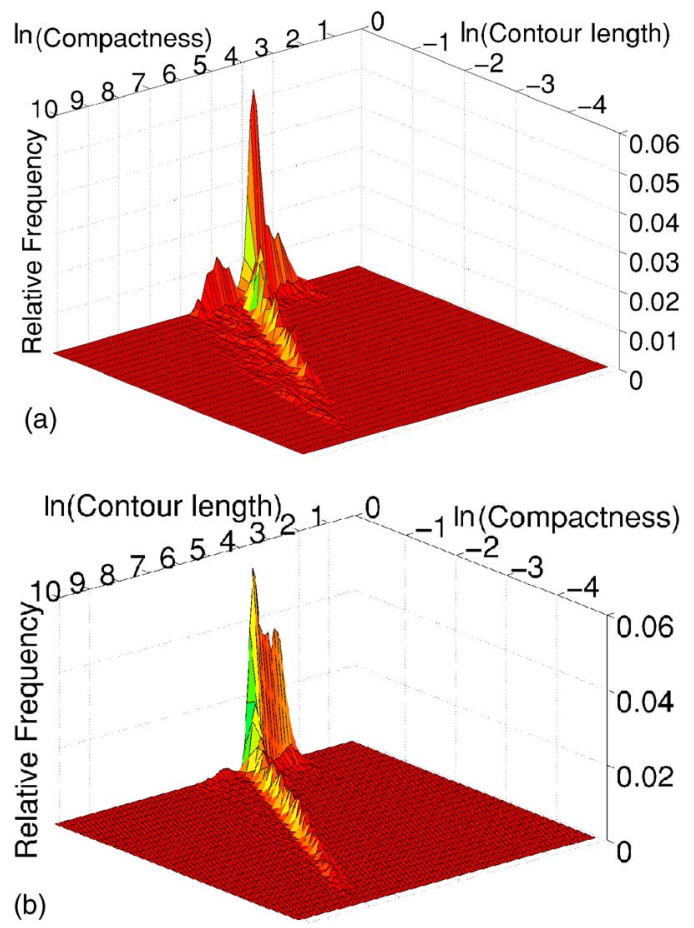

FIG. 14. (Color online) Distribution function for contour length and compactness for $\mathrm{OB}$ (a) and $\mathrm{NB}$ conditions (b). $\mathrm{Pr}=0.8$ and $\epsilon$ $=1.4$.

snapshot of the NB case Fig. 9(b). Thus, the comparably high relative frequency of small components shown in Fig. 13 has a different origin in the $\mathrm{OB}$ and the NB case. Whereas in the NB case it is mostly due to small bubbles, it seems to originate from targetlike structures in the $\mathrm{OB}$ case.

In the logarithmic scaling used in Fig. 14 a straight ridge arises in the distribution function for large contour lengths. It is characteristic for filamentary structures with a typical width, which corresponds here to one-half a wavelength $\lambda$ of the convection rolls,

$$
\mathcal{C} \sim 4 \pi \frac{\lambda \mathcal{P}}{4 \mathcal{P}^{2}} \propto \mathcal{P}^{-1}
$$

In the $\mathrm{OB}$ case one can discern deviations from this scaling. They are confined to larger rather than smaller compactness values for a given contour length, indicating that the long rolls can be wider but not narrower than a certain thickness. In the NB case these deviations are much smaller.

To identify spiral components in the pattern directly we also measure the winding number of the components [27]. It is defined via the angle $\theta$ by which the (spiral) arm of a pattern component is rotated from its tip to its end at the vertex at which it merges with the rest of the component. In cases in which a component has no vertices we split it into two arms at the location of minimal curvature [27]. The winding number is then defined as $|\mathcal{W}|=\theta / 2 \pi$. To assess the impact of the NB effects on the spiral character of the pattern we measure the number of spirals in each snapshot and show the resulting histogram over the whole run in Fig. 15. We use three different thresholds $\mathcal{W}_{\text {min }}$ for the identification of spi- 


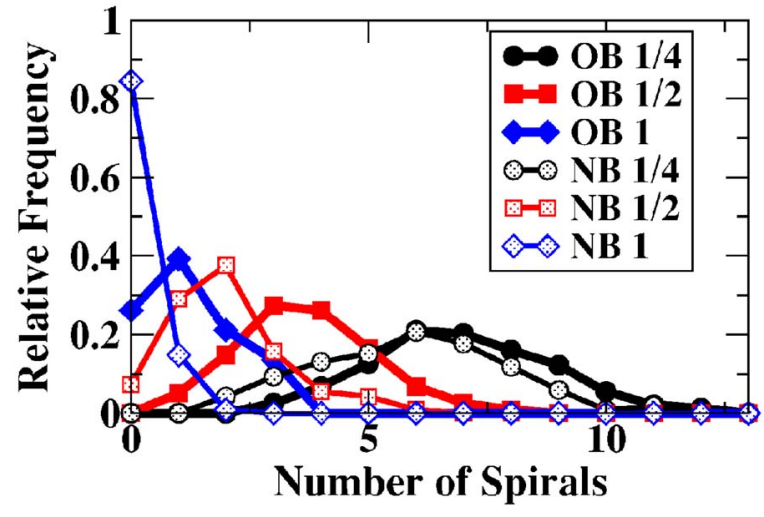

FIG. 15. (Color online) Distribution function for the number of spirals per snapshot in spiral defect chaos under OB and NB conditions for three values of the threshold, $\mathcal{W}_{\min }=1 / 4$ (circles), $\mathcal{W}_{\min }=1 / 2$ (squares), and $\mathcal{W}_{\min }=1$ (diamonds).

rals, $\mathcal{W}_{\min }=1, \mathcal{W}_{\min }=1 / 2$, and $\mathcal{W}_{\min }=1 / 4$. As Fig. 15 shows, the number of small spirals with $|\mathcal{W}| \gtrsim 1 / 4$ is quite similar in the $\mathrm{OB}$ and the NB case. However, larger spirals with $|\mathcal{W}| \geq 1 / 2$ or even $|\mathcal{W}| \geq 1$ are much more rare in the NB case; in fact, for the system size $L=16 \times 2 \pi / q_{c}=32.22$ that we have used in these simulations there was at most one spiral with $|\mathcal{W}| \geq 1$ at any given time.

The reduced spiral character of NB spiral defect chaos is also quite apparent in Fig. 16, which shows the correlation between the winding number and the arc length of the spiral arm. More precisely, each dot marks the occurrence of one spiral arm in a snapshot. In the $\mathrm{OB}$ case one can see quite clearly a maximal winding number for any given arc length, which is consistent with an Archimedean shape of the spiral $[27,59,56]$. In the NB case, however, only components with very small contour length reach the Archimedean limit and most components have winding numbers that are much smaller, i.e., the components are quite straight.

\section{HEXAGONS AND SPIRAL DEFECT CHAOS AT VERY LOW PRANDTL NUMBERS: $\mathrm{H}_{2}$-XeMIXTURES}

As mentioned above, the restabilization of NB hexagons at larger Rayleigh number is related to the existence of stable OB hexagons at large Rayleigh numbers. The wave number range over which the $\mathrm{OB}$ hexagons are stable shrinks with

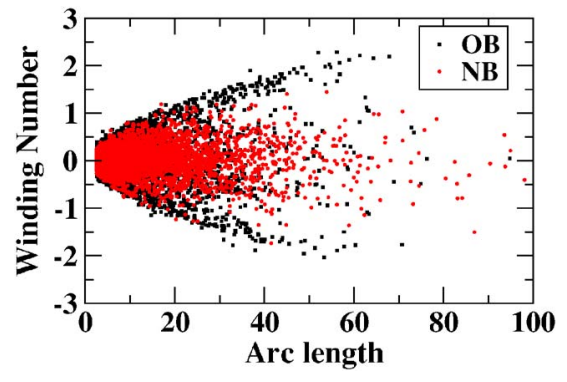

FIG. 16. (Color online) Correlation between the arc length and the winding number of spiral arms under OB (black squares) and $\mathrm{NB}$ conditions (red circles).

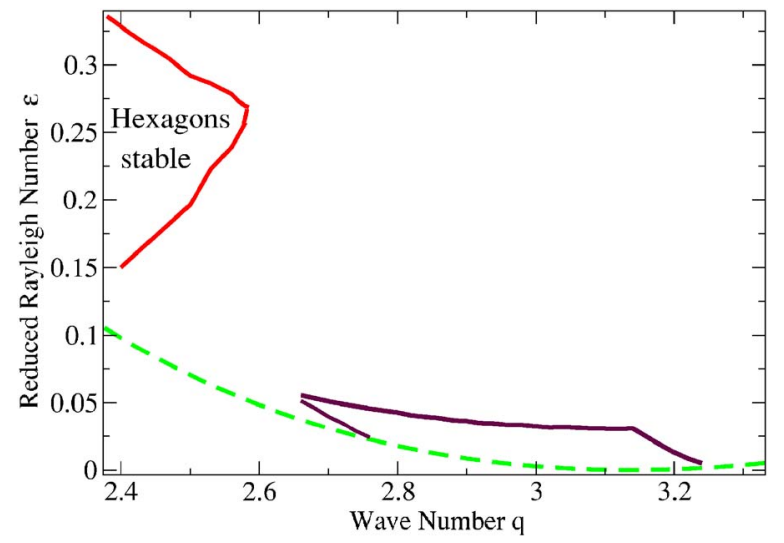

FIG. 17. (Color online) Stability limits with respect to side-band perturbations in a mixture of $\mathrm{H}_{2}-\mathrm{Xe}$ with molar fraction $\chi=0.4$, thickness $d=0.1 \mathrm{~cm}$, pressure $P=300 \mathrm{psi}$, mean temperature $T_{0}$ $=80^{\circ} \mathrm{C}$, and Prandtl number $\operatorname{Pr}=0.17$. The NB coefficients are $\gamma_{0}$ $=0.5535, \gamma_{1}=-0.6421, \gamma_{2}=0.9224, \gamma_{3}=0.3647, \gamma_{4}=-0.0712$ resulting in $Q=13.8$. Hexagons are stable in the regions enclosed by the solid lines and unstable outside. The dashed line represents the neutral curve. The results for $\mathrm{H}_{2}$-Xe are obtained with $n_{z}=6$ and $n_{q}$ $=5$.

decreasing Prandtl number and for $\operatorname{Pr}<1.2$ the OB hexagons are side-band unstable at all wave numbers [31]. However, as seen in the case of $\mathrm{CO}_{2}$ and $\mathrm{SF}_{6}, \mathrm{NB}$ hexagons can be side-band stable at large $\epsilon$ even below $\operatorname{Pr}=1.2$ due to the additional stabilizing effect of the resonant triad interaction. It is therefore of interest to investigate whether the NB effects can be sufficient to stabilize strongly nonlinear hexagons even for Prandtl numbers significantly below $\operatorname{Pr}=1$.

Prandtl numbers well below $\operatorname{Pr}=1$ can be reached by using a mixture of a heavy and a light gas. An experimentally investigated case is a mixture of $\mathrm{H}_{2}$ and $\mathrm{Xe}$ [41]. With a mole fraction of $\chi=0.4$, one can reach Prandtl numbers as small as $\operatorname{Pr}=0.17$. The Lewis number of such a mixture is close to one $[19,42]$. Therefore such mixtures are expected to behave essentially like a pure fluid with the same Prandtl number [1].

We investigate the stability of hexagon and roll convection in a $\mathrm{H}_{2}$-Xe mixture with mole fraction $\chi=0.4$ at a pressure of 300 psi and a layer thickness of $d=0.1 \mathrm{~cm}$. With respect to amplitude instabilities the stability diagram is very similar to that of convection in $\mathrm{CO}_{2}$ and $\mathrm{SF}_{6}$ with hexagons becoming reentrant at $\epsilon$ values as low as $\epsilon=0.14$ for $T_{0}$ $=20{ }^{\circ} \mathrm{C}$.

Focusing on strong NB effects we perform a detailed stability analysis with respect to side-band perturbations at a mean temperature of $T_{0}=80{ }^{\circ} \mathrm{C}$ using the same layer thickness of $d=0.1 \mathrm{~cm}$. For these low Prandtl numbers the numerical resolution must be increased to obtain sufficiently well resolved flows. While for the stability anlyses of hexagons in $\mathrm{CO}_{2}$ and $\mathrm{SF}_{6}$ it is sufficient to use $n_{z}=6$ and $n_{q}=3$ in the Galerkin expansion, for the $\mathrm{H}_{2}$-Xe mixture with $\mathrm{Pr}$ $\simeq 0.17$ at least $n_{q}=5$ and $n_{z}=6$ are required. Figure 17 depicts the resulting stability diagram. It shows that the region of side-band stable hexagons is not contiguous but consists of the usual region immediately above threshold and an additional, disconnected region at larger Rayleigh numbers. We 


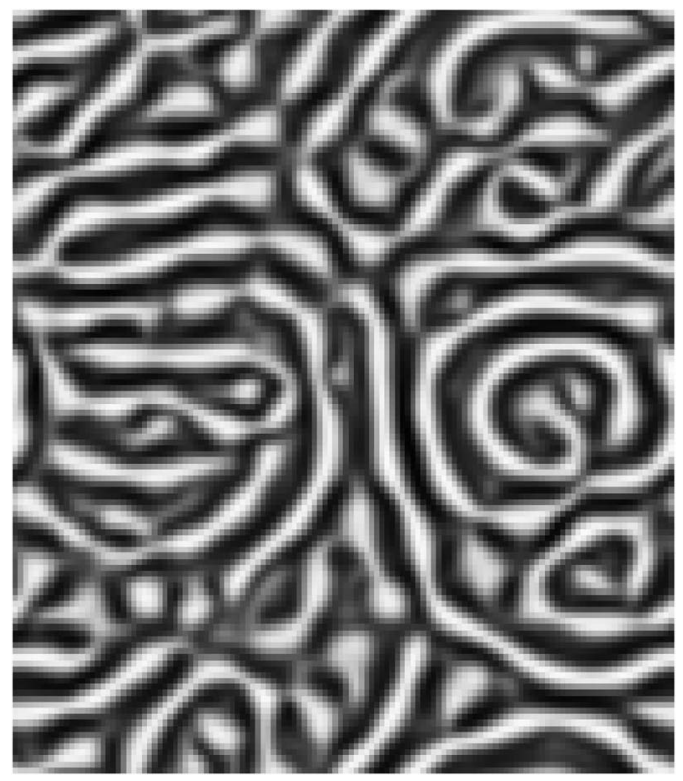

FIG. 18. Typical pattern for convection in $\mathrm{H}_{2}-\mathrm{Xe}$ at $\epsilon=0.3$.

could not follow the stability limits to smaller values of $q$ than shown in Fig. 17 due to numerical convergence problems. Presumably, these arise due to bifurcations involving additional, resonant wave vectors [43], somewhat similar to the resonances studied in Taylor vortex flow $[44,45]$. The fact that the region of stability is disconnected is remarkable since the region of amplitude stability (not shown) is actually contiguous. This is in contrast to the behavior found in $\mathrm{CO}_{2}$ and $\mathrm{SF}_{6}$ where the two side-band stable regions become connected when the bubblelike region of amplitude instability disappears (cf. Figs. 2 and 3). The comparison of the stability limits with those of $\mathrm{CO}_{2}$ and of $\mathrm{SF}_{6}$ [Fig. 8(b)] shows further that the maximal wave number $q$ at which the hexagons are stable with respect to side-band perturbations decreases with decreasing Prandtl number and as a result the overall stability region shrinks as well.

The stability analysis of the $\mathrm{H}_{2}$-Xe mixture also reveals an oscillatory instability of the hexagon patterns at $\epsilon \sim 1$. Within the $\epsilon$-range investigated, no such oscillatory instability was found at the larger Prandtl numbers relevant for $\mathrm{CO}_{2}$ and $\mathrm{SF}_{6}$. Unfortunately, it turns out that before the onset of the oscillatory instability the hexagons already become unstable to a side-band instability at one-half the hexagon wavelength, which will always preempt the oscillatory instability. For the rolls we also find an oscillatory instability. It is presumably related to the well-known oscillatory instability of Boussinesq rolls [46].

For not too small $\epsilon$ generic initial conditions will not lead to hexagonal patterns but rather to spiral defect chaos. A typical snapshot of a pattern in this state is shown in Fig. 18 for $\epsilon=0.3$. Compared to the patterns obtained in NB convection in $\mathrm{SF}_{6}$ [cf. Fig. 9(b)] the patterns in $\mathrm{H}_{2}$-Xe are less cellular and do not show a large number of small bubbles. To quantify these and other characteristics of the patterns we again apply the geometric diagnostics introduced earlier [27].

In Fig. 19 we show the normalized distribution functions for the compactness of white and black components (cf. Fig.

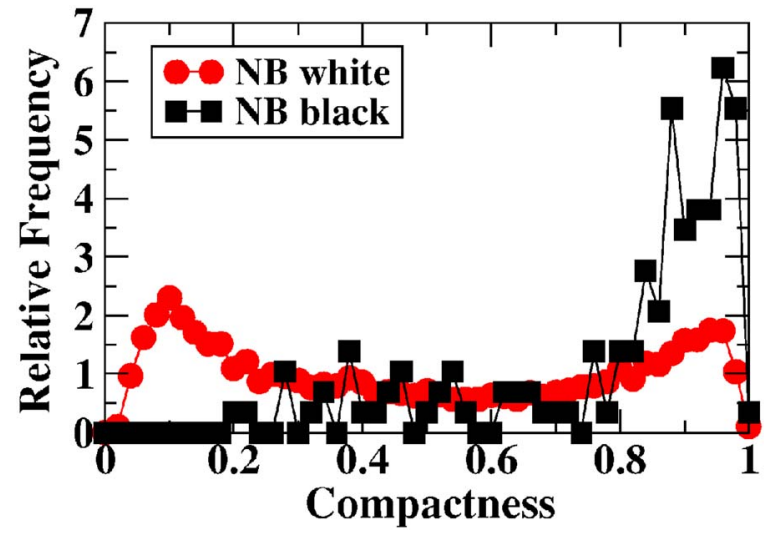

FIG. 19. (Color online) Normalized distribution function of the compactness of closed contours for $\mathrm{H}_{2}-\mathrm{Xe}(\mathrm{Pr}=0.17, \epsilon=0.3)$.

13). Since there are only very few black components their distribution function exhibits large statistical fluctuations. Of particular interest is the distribution function for the white components. It confirms the visual impression that the number of compact components is significantly reduced compared to the case of $\mathrm{SF}_{6}$; in fact, while in $\mathrm{SF}_{6}$ the maximum of the distribution function is close to $\mathcal{C}=1$, in $\mathrm{H}_{2}-\mathrm{Xe}$ the absolute maximum is at $\mathcal{C} \sim 0.1$, which corresponds to filamentary structures.

The lack of small bubbles is demonstrated in more detail in the joint distribution function for the contour length and the compactness, which is shown in Fig. 20. Note that the view is rotated compared to Fig. 14. The distribution function is lacking the broad shoulder towards small contour lengths seen in $\mathrm{NB}$ convection in $\mathrm{SF}_{6}$ [see Fig. 14(b)]. Instead, the decay of the distribution function towards long filamentary contours is quite slow.

To assess the spiral character of NB spiral defect chaos at these low Prandtl numbers we show in Fig. 21 the distribution function for the absolute value $|\mathcal{W}|$ of the winding number for $\mathrm{NB}$ convection in $\mathrm{H}_{2}$ - $\mathrm{Xe}$ (at $\epsilon=0.3$ and $\mathrm{Pr}=0.17$ ) as well as for Boussinesq and non-Boussinesq convection in $\mathrm{SF}_{6}$ (at $\epsilon=1.4$ and $\operatorname{Pr}=0.8$ ). As had been noted previously in the Boussinesq case $[27,47]$ the distribution function is

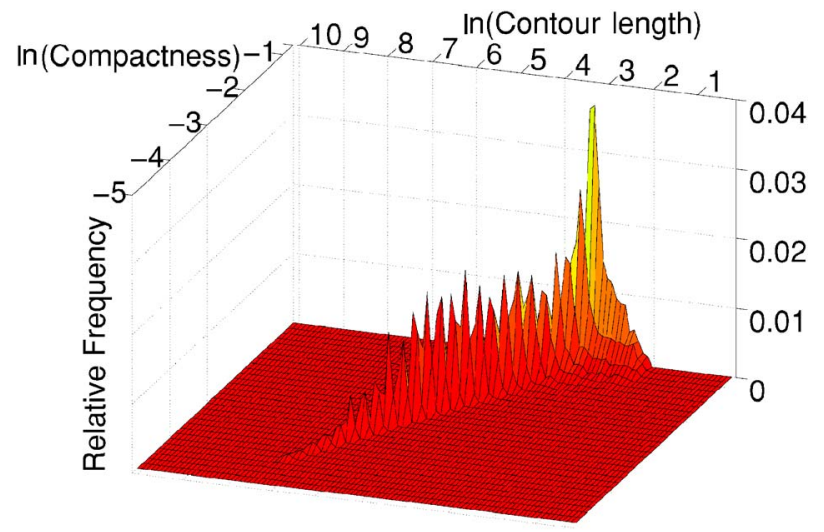

FIG. 20. (Color online) Joint distribution function for the contour length and the compactness of closed contours for $\mathrm{H}_{2}-\mathrm{Xe}(\mathrm{Pr}$ $=0.17, \epsilon=0.3)$. 


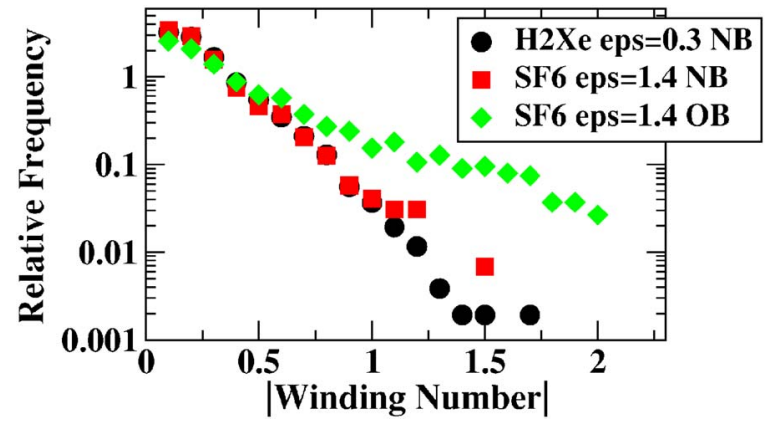

FIG. 21. (Color online) Normalized distribution function for the absolute value of the winding number for $\mathrm{NB}$ convection in $\mathrm{H}_{2}-\mathrm{Xe}$ in comparison with convection in $\mathrm{SF}_{6}$ in the $\mathrm{NB}$ and the $\mathrm{OB}$ case.

roughly consistent with exponential behavior. In the NB case the exponential decays substantially faster than in the Boussinesq case and spirals with winding numbers above $|\mathcal{W}|=1$ are rare. In the Boussinesq case we had found that the decay rate depends mostly on the Prandtl number, but only very little on $\epsilon$ [27]. Unfortunately, we do not have enough nonBoussinesq data to investigate such trends in the $\epsilon$ and $\operatorname{Pr}$ dependence. However, it is worth noting that in the two nonBoussinesq cases shown in Fig. 21 the decay rates are essentially the same despite their substantial difference in Prandtl number and both decays are much faster than that in the Boussinesq case. Thus, possibly the impact of NB effects dominates the dependence on the Prandtl number.

\section{CONCLUSION}

In this paper we have studied non-Boussinesq convection in gases $\left(\mathrm{CO}_{2}, \mathrm{SF}_{6}, \mathrm{H}_{2}-\mathrm{Xe}\right)$ with Prandtl numbers ranging from $\operatorname{Pr} \sim 1$ down to $\operatorname{Pr}=0.17$ in experimentally relevant parameter regimes. We have complemented a Galerkin stability analysis of hexagon patterns with direct numerical simulations of the fluid equations to study transitions between different hexagon patterns and to quantify the impact of nonBoussinesq effects on spiral defect chaos.

We find that the reentrance of hexagons that we have identified previously in non-Boussinesq convection in water [16] also occurs at low Prandtl numbers. As was the case at large Prandtl numbers, compressibility is not necessary for reentrance. Since, in addition, the range of wave numbers for which the reentrant hexagons are stable differs significantly from that of the reentrant hexagons observed in experiments on convection in $\mathrm{SF}_{6}$ near the thermodynamic critical point [15], the mechanisms underlying the two types of restabilization of the hexagons are most likely different. Reflecting the fact that in gases the non-Boussinesq effects increase with increasing temperature the reentrance is shifted to lower values of the Rayleigh number when the mean temperature is increased, opposite to the behavior in water [16]. In convection in water the reentrant hexagons are stable only for wave numbers below the critical wave number. This trend becomes more pronounced with decreasing Prandtl number. In fact, for the gas mixture with $\operatorname{Pr}=0.17$ the wave number at the stability limit of the hexagons decreases so rapidly with increasing Rayleigh number that the range in Rayleigh number over which the hexagons are stable becomes quite small.

Our stability analysis confirms the weakly nonlinear prediction [26] that for sufficiently small Prandtl numbers the usual transverse long-wave instabilities of hexagons is replaced by the longitudinal one. Our numerical simulations also show the expected difference in the complexity of the ensuing transients.

The comparison of our stability results with experiments on the transition between hexagons and rolls in $\mathrm{CO}_{2}$ [12] shows that this transition is not due to an amplitude or a side-band instability. As a matter of fact, for the parameters of the experimental system the hexagons do not undergo any linear amplitude instability to rolls, contrary to the prediction of the weakly nonlinear theory [12]. We have performed detailed numerical simulations with various lateral boundary conditions and confirm that the transition is the result of the heterogeneous nucleation of rolls at the side walls of the container, which then invade the whole system if the Rayleigh number is sufficiently high. Our simulations suggest that hexagons could be stabilized well beyond the experimentally observed transition point if the influence of the lateral walls can be reduced by applying a spatially patterned forcing that drives hexagons at the wall. Such a forcing can be achieved by localized heating [35] or by geometric patterning of the bottom plate [36]. Of course, the wave number of the forced hexagons would have to be adjusted to lie in the stable range.

We have also investigated the stability of hexagons in $\mathrm{H}_{2}$-Xe mixtures with very small Prandlt number $(\mathrm{Pr}=0.17)$. There also stable reentrant hexagons are possible, but they are restricted to a small range in wave number $\left(q<q_{c}\right)$ and Rayleigh number. Since for such small Prandtl numbers Boussinesq hexagons are always side-band unstable [31], the stability of the hexagons is a result of the non-Boussinesq effects.

We have not addressed the effect of the mean flow on non-Boussineseq convection in rotating systems. Within the Ginzburg-Landau framework it was found that mean flow can decrease the stability of penta-hepta defects [48]. In simulations the destabilization of penta-hepta defects manifested itself in the nucleation of a dislocation pair nearby, which then recombined with the two dislocations making up the penta-hepta defect. This induced defect nucleation was found to be the origin of a persistent chaotic state [48,49]. An interesting question is, of course, whether these phenomena can also be obtained in the full Navier-Stokes equations.

A fascinating state that is characteristic for convection at low Prandtl numbers is spiral defect chaos [17]. We have studied the influence of NB effects on spiral defect chaos in a setup corresponding to convection in $\mathrm{SF}_{6}$ with a Prandtl number of $\mathrm{Pr}=0.8$ and in $\mathrm{H}_{2}$-Xe with a Prandtl number of $\operatorname{Pr}=0.17$. To quantify the differences between Boussinesq and non-Boussinesq spiral defect chaos we have employed a recently suggested set of geometric diagnostics of the patterns [27]. In contrast to global measures like correlation functions and spectral entropy [50] and to measures that are sensitive to the curvature and the deformation of a regular pattern [51,52], our measures extract local features of the patterns, which can be quite disordered. Our diagnostics are aimed at characterizing chiral aspects of patterns like spirals 
and at distinguishing lamellar from cellular patterns. Thus, they do not assume that the patterns are locally stripelike [53-55].

As expected, in $\mathrm{SF}_{6}$ and in $\mathrm{H}_{2}$-Xe the non-Boussinesq effects break the equivalence of up and down flows and, consequently, the mean number of pattern components (closed contours) corresponding to down flow differs significantly from that of the up-flow components. More interesting is our finding that in $\mathrm{SF}_{6}$ the total number of components (Betti number [39]) is more than 2 times as large in the non-Boussinesq case than in the Boussinesq case for otherwise equal parameters. We attribute this enhancement of cellular rather than roll-like structures to the resonant triad interaction that is introduced by the non-Boussinesq effects.

Another striking difference between Boussinesq and nonBoussinesq spiral defect chaos in $\mathrm{SF}_{6}$ becomes apparent in the joint distribution function for the contour length of the components and their compactness. While the Boussinesq case exhibits a strong signature of targetlike structures, the non-Boussinesq case displays instead a marked increase in the number of small compact components (bubbles). This trend towards a more cellular structure is also apparent in the analysis of spirals. The number of spirals with a winding number above $\frac{1}{2}$ is much smaller in the non-Boussinesq than in the Boussinesq case. In the correlations between the winding number and the arc length of the components this is reflected by the missing of large Archimedean spirals in the non-Boussinesq case.

Interestingly, we do not find many small bubbles in our simulations of $\mathrm{H}_{2}$-Xe at $\mathrm{Pr}=0.17$. In fact, the maximum of the distribution function for the compactness of pattern components is shifted away from $\mathcal{C} \leq 1$ to $\mathcal{C} \sim 0.1$, i.e., to filamentary structures. We do not know whether this implies that the tendency towards small bubbles is maximal at moderately small Prandtl numbers, i.e., for $\operatorname{Pr} \sim 1$, or whether it is due to the fact that for computational reasons the simulations in $\mathrm{H}_{2}$-Xe were performed at a lower Rayleigh number ( $\epsilon$ $=0.3)$ than in $\mathrm{SF}_{6}(\epsilon=1.4)$.

Due to computational limitations we have not been able to investigate the transition from hexagons to spiral defect chaos at very small Prandtl numbers. Since for very small Prandtl numbers there are essentially no large spirals, the transition from hexagons to spiral defect chaos may follow a different path than that observed experimentally in $\mathrm{CO}_{2}$, where large spirals were reported to arise in the transition from hexagons to rolls [12]. We surmise that the geometric diagnostics that we have employed to characterize the well developed spiral defect chaos would provide also valuable insight into this transition. Since the diagnostics require a substantial amount of data the transition would be best investigated experimentally.

\section{ACKNOWLEDGMENTS}

The authors deeply appreciate the ongoing support by W. Pesch, who together with his students has developed the codes we have used in this study [22]. The authors thank G. Ahlers for providing the code to determine the NB coefficient. The authors have benefitted from discussions with G. Ahlers, G. Gunaratne, K. Krishan, K. Mischaikow, W. Pesch, and M. Schatz. Support from the Department of Energy Grant No. (DE-FG02-92ER14303), NSF Grant No. (DMS0309657), and by EU under Grant No. MRTN-CT-2004005728 [to one of the authors (S. M.)] is gratefully acknowledged.
[1] E. Bodenschatz, W. Pesch, and G. Ahlers, Annu. Rev. Fluid Mech. 32, 709 (2000).

[2] F. H. Busse, J. Fluid Mech. 30, 625 (1967).

[3] E. Palm, J. Fluid Mech. 8, 183 (1960).

[4] L. A. Segel and J. T. Stuart, J. Fluid Mech. 13, 289 (1962).

[5] L. A. Segel, J. Fluid Mech. 21, 359 (1965).

[6] E. Palm, T. Ellingsen, and B. Gjevik, J. Fluid Mech. 30, 651 (1967).

[7] S. H. Davis and L. A. Segel, Phys. Fluids 11, 470 (1968).

[8] E. F. Somerscales and T. S. Dougherty, J. Fluid Mech. 42, 755 (1970).

[9] M. Dubois, P. Bergé, and J. Wesfreid, J. Phys. (France) 39, 1253 (1978).

[10] F. M. Richter, J. Fluid Mech. 89, 553 (1978).

[11] R. W. Walden and G. Ahlers, J. Fluid Mech. 109, 89 (1981).

[12] E. Bodenschatz, J. R. deBruyn, G. Ahlers, and D. S. Cannell, Phys. Rev. Lett. 67, 3078 (1991).

[13] E. Pampaloni, C. Pérez-Garía, L. Albavetti, and S. Ciliberto, J. Fluid Mech. 234, 393 (1992).

[14] M. Assenheimer and V. Steinberg, Phys. Rev. Lett. 76, 756 (1996).

[15] A. Roy and V. Steinberg, Phys. Rev. Lett. 88, 244503 (2002).
[16] S. Madruga, H. Riecke, and W. Pesch, J. Fluid Mech. 548, 341 (2006).

[17] S. W. Morris, E. Bodenschatz, D. S. Cannell, and G. Ahlers, Phys. Rev. Lett. 71, 2026 (1993).

[18] S. W. Morris, E. Bodenschatz, D. S. Cannell, and G. Ahlers, Physica D 97, 164 (1996).

[19] J. Liu and G. Ahlers, Phys. Rev. Lett. 77, 3126 (1996).

[20] H. W. Xi, J. D. Gunton, and J. Viñals, Phys. Rev. Lett. 71, 2030 (1993).

[21] M. C. Cross and Y. Tu, Phys. Rev. Lett. 75, 834 (1995).

[22] W. Decker, W. Pesch, and A. Weber, Phys. Rev. Lett. 73, 648 (1994).

[23] K.-H. Chiam, M. R. Paul, M. C. Cross, and H. S. Greenside, Phys. Rev. E 67, 056206 (2003).

[24] Y.-N. Young, H. Riecke, and W. Pesch, New J. Phys. 5, 135 (2003).

[25] S. Madruga and C. Pérez-García, Int. J. Bifurcation Chaos Appl. Sci. Eng. 14, 107 (2004).

[26] Y.-N. Young and H. Riecke, Physica D 163, 166 (2002).

[27] H. Riecke and S. Madruga, Chaos 16, 013125 (2006).

[28] F. H. Busse and R. M. Clever, J. Fluid Mech. 91, 319 (1979).

[29] S. Chandrasekhar, Hydrodynamic and Hydromagnetic Stability 
(Clarendon, Oxford, 1961).

[30] W. Pesch, Chaos 6, 348 (1996).

[31] R. M. Clever and F. H. Busse, Phys. Rev. E 53, R2037 (1996).

[32] W. Decker and W. Pesch, J. Phys. II 4, 419 (1994).

[33] A. Bernoff, Eur. J. Appl. Math. 5, 267 (1994).

[34] M. Sushchik and L. Tsimring, Physica D 74, 90 (1994).

[35] D. Semwogerere and M. F. Schatz, Phys. Rev. Lett. 88, 054501 (2002).

[36] E. Bodenschatz (private communication).

[37] E. Bodenschatz, D. Cannell, J. R. DeBruyn, R. Ecke, Y. Hu, K. Lerman, and G. Ahlers, Physica D 61, 77 (1992).

[38] M. Assenheimer and V. Steinberg, Nature (London) 367, 345 (1994)

[39] M. Gameiro, K. Mischaikow, and W. Kalies, Phys. Rev. E 70, 035203(R) (2004).

[40] K. Krishan, M. Gameiro, K. Mischaikow, and M. F. Schatz, arxiv.org/abs.nlin.PS/0701043 (unpublished).

[41] J. Liu and G. Ahlers, Phys. Rev. E 55, 6950 (1997).

[42] G. Ahlers, in Dynamics of Spatio-Temporal Cellular Structures - Henri Bénard Centenary Review, edited by I. Mutabazi, J. E. Wesfreid, and E. Guyon, Springer Tracts in Modern Physics (Springer, New York, 2005).

[43] V. V. Moroz, Ph.D. thesis, Northwestern University, 2004.

[44] H. Riecke and H.-G. Paap, Phys. Rev. A 33, 547 (1986).

[45] H.-G. Paap and H. Riecke, Phys. Rev. A 41, 1943 (1990).

[46] V. Croquette and H. Williams, Phys. Rev. A 39, R2765 (1989).
[47] R. Ecke and Y. Hu, Physica A 239, 174 (1997).

[48] Y.-N. Young and H. Riecke, Physica D 176, 107 (2003).

[49] Y.-N. Young and H. Riecke, Phys. Rev. Lett. 90, 134502 (2003).

[50] R. V. Cakmur, D. Egolf, B. B. Plapp, and E. Bodenschatz, Phys. Rev. Lett. 79, 1853 (1997).

[51] G. H. Gunaratne, R. E. Jones, Q. Ouyang, and H. L. Swinney, Phys. Rev. Lett. 75, 3281 (1995).

[52] S. Hu, G. Nathan, D. Kouri, D. Hoffman, and G. Gunaratne, Chaosthe 15043701 (2005).

[53] D. A. Egolf, I. V. Melnikov, and E. Bodenschatz, Phys. Rev. Lett. 80, 3228 (1998).

[54] M. Cross, D. Meiron, and Y. Tu, Chaos 4, 607 (1994).

[55] Y. Hu, R. Ecke, and G. Ahlers, Phys. Rev. E 51, 3263 (1995).

[56] B. Plapp, Ph.D. thesis, Cornell University, 1997.

[57] These values were obtained with a code kindly provided by Ahlers.

[58] In the experimental setup the top temperature is held constant at $T=12.84{ }^{\circ} \mathrm{C}$, and therefore the mean temperature changes as $\epsilon$ is increased. In our computations, however, we keep $T_{0}$ fixed. Since the transition occurs quite close to threshold this is a good approximation to the experimental procedure.

[59] Note that detailed analyses of large spirals shows deviations from the Archimedean shape due to the dislocations that accompany finite spirals [56]. 